\title{
Assessing the epigenetic risks of assisted reproductive technologies: a way forward
}

\author{
FEDERICA ZACCHINI ${ }^{1}$, SILVESTRE SAMPINO ${ }^{2}$, ADRIAN M. STANKIEWICZ ${ }^{2}$, THOMAS HAAF ${ }^{3}$ and \\ GRAZYNA E. PTAK*,1 \\ ${ }^{1}$ Małopolska Centre of Biotechnology, Jagiellonian University, Kraków, Poland \\ ${ }^{2}$ Institute of Genetics and Animal Breeding of the Polish Academy of Sciences, Jastrzebiec, Poland and \\ ${ }^{3}$ Institute of Human Genetics, Julius Maximillians University, Würzburg, Germany
}

\begin{abstract}
Since the birth of the first baby conceived by in vitro fertilization (IVF), assisted reproductive technologies (ART) have been constantly evolving to accomodate needs of a growing number of infertile couples. Rapidly developing ART procedures are directly applied for human infertility treatment without prior long-term safety evaluation. Although the majority of ART babies are healthy at birth, a comprehensive assessment of the long-term risks associated with ART is still lacking. An increased risk of epigenetic errors has been associated with the use of ART, which may contribute to the onset of civilization disease later in adolescence/adulthood and/or in subsequent generations. Therefore, our investigations should not focus on (or be limited to) the occurrence of a few very rare imprinting disorders in ART children, which might be associated with parental age and/or the use of ART, but on the possibly increased disease susceptibilities later in life and their potential transmission to the subsequent generations. Retrospective studies do not offer exhaustive information on long-term consequences of ART. Animal models are useful tools to study long-term effects including transgenerational ones and the epigenetic risk of a given ART procedure, which could then be translated to the human context. The final goal is the establishment of common guidelines for assessing the epigenetic risk of ART in humans, which will contribute to two key objectives of the Horizon2020 programme, i.e. to improve our understanding of the causes and mechanisms underlying health and disease, and to improve our ability to monitor health and prevent/manage disease.
\end{abstract}

KEY WORDS: ART, epigenetics, placentae

\section{Introduction}

Safe use of ART remains an object of debate (Brison et al., 2013; Harper et al., 2014, 2015). To provide exhaustive answers is not easy due to continuous changes in ART practice (i.e. new media, supplements, incubation conditions) and to numerous confounding factors (e.g. parental age, male or female infertility, social status, lifestyle, concomitant maternal pathologies) that may be associated with a pregnancy loss and/or other health complications. The complexity and invasiveness of ART procedures is increasing. Multiple procedures, such as three biopsies plus double cryopreservation performed on the same embryo (Greco et al., 2015), were recently introduced into a clinical setting, without proper testing before their application. The most aggressive procedure ever applied in human ART, consisting of the transfer of the Metaphase II (MII) plate or pronuclei from low-quality oocytes/zygotes into enucleated oocytes/ zygotes from young healthy donors, has been introduced in UK ("three parents babies", Amato et al., 2014). Relevant knowledge from animal models is still very preliminary.

The Developmental Origin of Health and Disease (DOHAD) hypothesis asserts that the perturbation of key events during the periconceptional period and/or early development leads to an increased risk of chronic diseases later in life (Barker et al., 1989: Heidel et al., 2015). Different ART coincide with this very sensitive developmental window. Adverse early life exposures, resulting

Abbreviations used in this paper: ART, assisted reproductive technologies; DOHAD, developmental origin of heath and diseases; DNMT1, DNA methyltrasferase 1; ICSI, intra cytoplasmatic sperm injection; IVM, in vitro maturation; IVF, in vitro fertilization, MII, metaphase II.

\footnotetext{
*Address correspondence to: Grazyna E. Ptak. Małopolska Centre of Biotechnology, Jagiellonian University, Kraków, Poland. e-mail: g.ptak@uj.edu.pl - (iD) https://orcid.org/0000-0003-1437-4698
} 
in altered epigenetic marks (epimutations), increase the risk of developing metabolic, cardiovascular, neuropsychiatric and other complex diseases later in life (Jiang et al., 2017; Chen and Zang, 2011; Meister et al., 2018). The term "epigenetic marks" indicates non-covalent modifications (or marks) of DNA or proteins (e.g. histones) that do not change DNA sequence per se but regulate gene expression in a quantitative and qualitative manner. Epigenetic mechanisms involve DNA and histone modification as well as microRNAinteractions and remodeling of chromatin complexes. DNA methylation is still the most thoroughly studied epigenetic marker for disease (Barlow, 2011; Sadakierska-Chudy and Filip, 2014). During development, epigenetic competence is acquired through two waves of epigenetic reprogramming: the first demethylationremethylation wave occurs during gametogenesis and the second during pre-implantation development (Reik and Dean, 2001b) (Fig. 1). In the male germline, sperm remethylation is already set before completion of meiosis and thus, less likely influenced by ART. On the other hand, paternal genome remodelling that occurs following fertilization (e.g. replacement of protamines with histones) might be affected by in vitro conditions and thus contribute to the onset of epigenetic alterations (Cantone and Fisher, 2013). On the other hand, male subfertility has been associated with alterations in sperm DNA methylation (Marques et al., 2008; Kobayashi et al., 2009; El Hajj et al., 2011). Clinically it is well known that male fertility (including sperm production, motility and genome integrity) declines with paternal age and, therefore, it is not unexpected that paternal age is also associated with sperm methylation alterations (Jenkins et al., 2014). In the female germline, remethylation takes place during late stages of oocyte development. In fact, some maternal imprints may only be fully established after fertilization (El-Maari et al., 2001). In this light, it is plausible to assume that the maternal genome is much more plastic and susceptible to ART during late oocyte development (IVM, superovulation) and/or early embryo development (embryo culture and physical manipulation), when genome-wide epigenetic reprogramming occurs (Reik et al., 2001a). The epigenomic plasticity of the somatic genome of the new individual is then steadily decreasing during prenatal and postnatal life (Gluckman et al., 2009). The exposure to adverse environmental factors in early life has remarkable influence on the developing organism, including the transmission of epimutation to the next generations (non-genetic transgenerational inheritance) (Reik et al., 2001a; Van Montfoort et al., 2012; Szyf, 2015).

The following sections focus on perspectives and limitations of the current research on ART in context of its impact on the embryo and offspring epigenetics.

\section{Human studies on the epigenetic risks of ART - future perspectives and limitations}

Exposure of the organism to adverse environmental factors at critical stages of early development, including preimplantation development, affects its later metabolism and health status (DOHAD) (Barker et al., 1989; Roseboom et al., 2006, Feuer et al., 2013). Dramatic environmental changes in the earliest possible time window occur during assisted reproduction (Feuer and Rinaudo, 2016). Thus, our main concern should not be whether preimplantation development is compromised or the occurrence of a few rare imprinting disorders is increased in ART children, but disease susceptibility in later life. Long-term follow up studies of the disease and health status of ART children, similar to the Dutch famine cohort are needed. The individuals exposed to maternal undernutrition in 1944 (and their children) are systematically followed up since the 1990s (Roseboom et al., 2006). In another follow up study concerning DOHAD, the effects of early life nutritional programming of health and disease are collected since 1950 from several generations of residents in Gambia (West

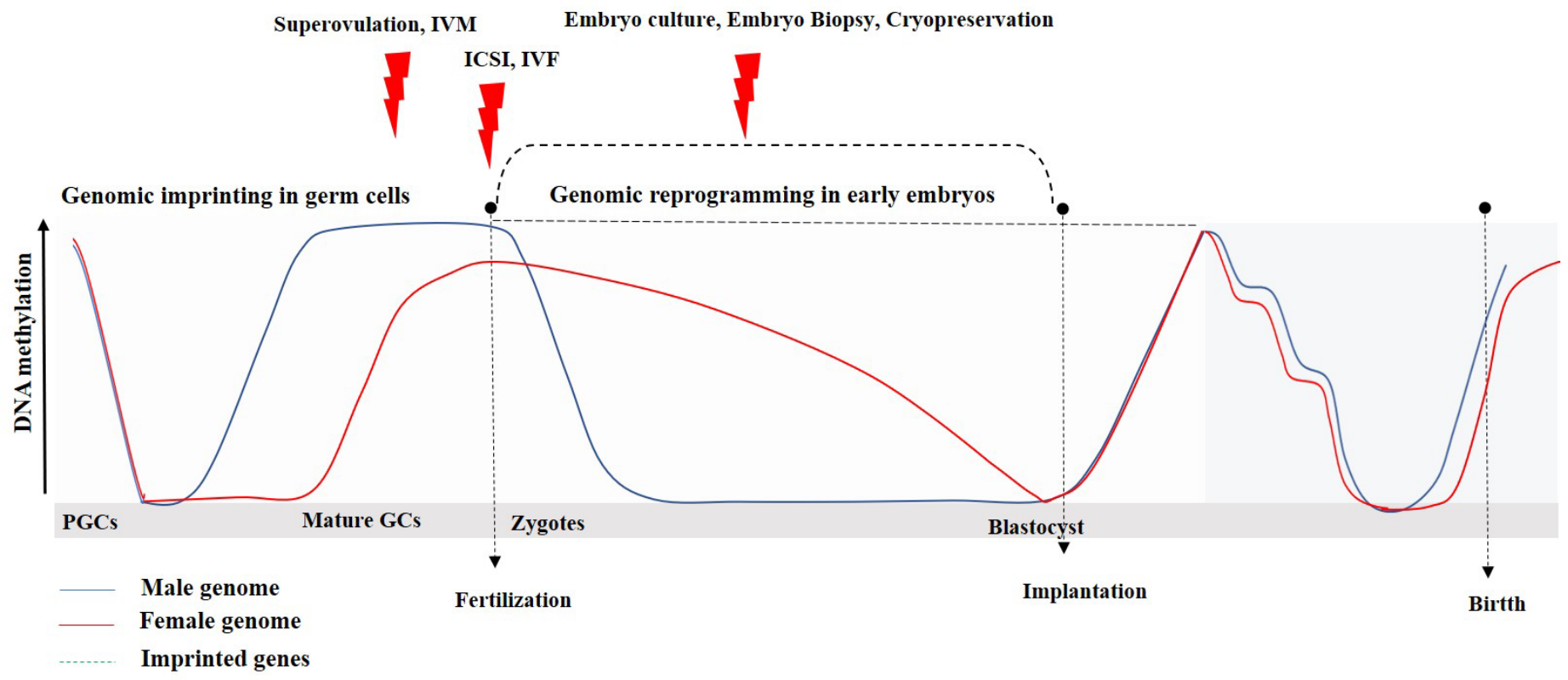

Fig. 1. Epigenetic genome reprogramming during gamete and early embryo development. The scheme shows i) epigenetic reprogramming of maternal (red) and paternal (blue) genomes in germ cells during gametogenesis, early embryonic and in utero development, and ii) sensitive periods of epigenetic reprogramming that may be affected by different Assisted Reproductive Technologies (ART). IVF, in vitro fertilization; ICSI, intracytoplasmatic sperm injection. Adapted from Rose et al., 2013 and Smallwood and Kelsey, 2012. 
Africa) (Moore, 2016).

Although over 5 million people have been conceived by IVF/ ICSI, most of them are still young and it will take several decades and systematic studies (which have not been implemented yet) to learn more about the long-term consequences of ART. In the last decade, it has been suggested that children born following ART might be at higher risk of cardiovascular diseases, metabolic (e.g. glucose metabolism perturbation) and psychiatric disorders (e.g. autism) and increased incidence of some type of cancer (e.g. acute lymphoblastic leukemia; retinoblastoma) (Vrooman and Bartolomei, 2017; Feuer et al., 2013; Meister et al., 2018). Transgenerational effects due to transmission of epigenetic information through the germline are even more difficult to assess. Transgenerational epigenetic inheritance has been well documented in plants, nematodes and flies, but its occurrence in mammals and particularly in humans remains controversial (Horsthemke, 2018).

So far, the main focus of human reproductive medicine has been on pregnancy rates and outcome at birth. Short-lived animals, such as rodents, provide ideal models to study the long-term consequences of different ART, which have been largely ignored until today. The placenta obtained at delivery is the best organ to identify epigenetic defects associated with future arousal of a given pathology (Choux et al., 2015). Even if epigenetic analysis (e.g. DNA methylation analysis of candidate genes or the entire genome) of full-term placentae cannot reduce the potential side effect of ART in the short term, it will be useful for developing therapeutic tools and, ultimately, improving ART procedures. In this context, the use of the placenta to assess epigenetic risk of ART is promising and is less limited compared to research on gametes and embryos. Furthermore, there are no ethical issues with the use of human placentas in such studies, as this organ is normally discarded at delivery. Moreover, it will be possible to obtain placental samples from both ART- and naturally-conceived/healthy pregnancies and, through the institution of tissue banks, perform large-scale studies. Another crucial point is that epigenomes are highly dynamic, varying between cell types/tissues and developmental stages. Studies on the epigenetic effects of different ART in humans are restricted to a few easily accessible tissues, such as placenta, cord blood or peripheral blood. However, the epigenetic changes mediating increased disease risks may only be present in the appropriate target tissue for a phenotype. For example, if an adverse effect increases metabolic disease risk, one should study the hypothalamic-pituitary-adrenal axis, fat, liver, skeletal muscle and/or pancreatic islets. This is only possible in the appropriate animal models.

One major problem in human studies is the enormous genetic and epigenetic heterogeneity. Epigenetic changes are at least one order of magnitude more frequent than genetic changes (BennettBaker et al., 2003). Epigenetic variation is shaped by sequence, gene-environment interactions and stochastic factors (Potabattula et al., 2018). There are many confounding factors influencing the human epigenomes, such as sex, age, nutrition, body mass index, alcohol consumption, smoking and other lifestyle factors. Consistent with a multifactorial disease model, epigenetic changes due to an adverse environmental exposure (e.g. during ART) usually have small effect sizes (at most in the order of several percentage points) and appear to be wide-spread. When studying human cohorts, i.e. ICSI children versus normally conceived children, even epigenetic markers which significantly differ between cohorts show a large overlap in the DNA methylation range between study subjects and controls (El Hajj et al., 2017). Thus, large sample sizes are needed to reach genome-wide significance. It is even more difficult to control and correct for all possible confounding factors. In genetically identical animals, which are kept under standardized conditions, the epigenetic noise is much lower and, therefore, it is more likely to attribute specific epigenetic effects to the defined environmental exposures, such as maturation of oocytes in different culture media, using different endpoints (e.g. matured oocytes, resulting embryos or animals).

Methylation analyses can be eithergenome-wide (i.e. methylation array, reduced representation or whole genome bisulfite sequencing) or locus-specific (i.e. bisulfite pyrosequencing, deep bisulfite sequencing). The common final goal of the different techniques is to provide methylation-based diagnostic tools that can identify sentinel loci and, in the long term, to develop new therapeutic options (Fazzari and Greally, 2010). In this light, it should be kept in mind that the interpretation of epigenetic data sets can be difficult. First of all, genome-wide studies produce huge amounts of data, thus it is necessary to perform accurate statistical analyses on specific loci or sets of loci and to associate differentially methylated regions with sequence composition. Second, though ART have been associated with increased risk of epigenetic defects (Odom and Segars, 2011; Anckaert et al., 2013; el Hajj and Haaf, 2013; Nelissen et al., 2013a), in humans it is not clear whether epigenetic changes, resulting in medical problems, are due to the ART procedures themselves or to parental factors. In the case of small epimutations, it is difficult to determine whether or not the epigenetic changes will lead to specific pathologic conditions in the short, medium or long term.

\section{Animal experiments in ART optimization}

So far all ART in humans have been introduced without a prior systematic evaluation of epigenetic risks. Consequently, techniques and media used in different laboratories/ART centers are not standardized, making multi-center studies necessary to increase the sample size difficult. Moreover, most companies do not even disclose the composition of widely used commercial media. Different culture conditions may lead to different pregnancy outcomes in terms of sex ratio and birth weight (Dumoulin et al., 2010; Zhu et al., 2014). Experiments in the Agouti mouse model convincingly showed that variation of a single factor (e.g. concentration of methyl donors) can have dramatic effects on the epigenome and the resulting phenotype (Waterland and Jirtle, 2003). When subsequently different human ART culture systems were tested in the mouse, a considerable variation in blastocyst and fetal development rates were noted (Schwarzer et al., 2012), while there were no gross morphological differences in the post implantation stages (Hemkemeyer etal., 2014). Recently, it has been shown that addition of natural reproductive fluids into culture medium reduced the occurrence of methylation defects as well as transcriptomic perturbation in in vitro produced pig embryos (Canovas et al., 2017). Due to species differences, animal experiments cannot be used for choosing the optimum medium for human embryo culture, but they can promote awareness and identify possible risk factors which should be carefully monitored. Most importantly, only animal models allow extensive organ analysis and rapid evaluation of health problems in $\mathrm{F} 1$ and/or further generations. 
Depending on the study question, the appropriate animal model can be chosen. For studying long-term effect (DOHAD), a model with short life-span is useful. However, in many aspects (e.g. oocyte and embryo development, gestation, parity), a bovine model is more similar to the human than rodents. Currently, studies on large animal models (sheep, bovine, horse, pig) are mainly focused on early development and pregnancy outcome. Available data showed epigenetic defects in early embryos and placenta and, in the later stage of development, increased pregnancy loss, altered placental structure and vascularization and increased birthweight, while little is known on the long-term health of the ART offspring in those models (see reviews, Duranton and Chavatte-Palmer, 2018; Bloise et al., 2014).

An animal model to test long-term impact of ART on the resulting offspring is an important clinical need. Animal studies could highlight potential adverse effect of a given procedure of an agent used in ART. If no such adverse effect were found, it would be reassuring for both medical practitioners and parents willing to conceive a baby. Embryos developed in vitro have different characteristics than those developed in vivo, as the in vitro environment will hardly overlap with physiological (oviductal and uterine) conditions (Rinaudo and Schultz, 2004; Market-Velker et al., 2010; Bertoldo et al., 2015). Recently, a cumulative effect of ART on a placental development/epigenetic profile has been described in mouse (de Waal et al., 2015). Similarly, we found that ART procedures - blastomere biopsy, in vitro embryo culture or simply embryo-transfer - affect global DNA methylation levels of murine fetal tissues collected at day 18 post coitum (Zacchini et al., 2018). All three ART procedures significantly decreased DNA methylation level in the placenta, while only embryo culture in vitro and blastomere biopsy had the same effect in the liver. Conversely, none of the procedures influenced global DNA methylation level in the brain. Interestingly, despite these global changes, DNA methylation profile of a very few specific genomic regions were significantly affected. We speculate that this phenomenon may be related to downregulation of DNMT1 mRNA expression in those embryos. We have previously reported that such downregulation occurs in early placenta obtained from sheep embryos cultured in vitro and then transferred to synchronized recipients (Ptak et al., 2013). DNMT1 enzyme mediates methylation of cytosines. It preferentially targets hemimethylated DNA and thus is responsible for maintenance of the methylation patterns during DNAreplication. Reduced activity of DNMT1 may cause accumulation of stochastic demethylation events between cell divisions. This is consistent with a stochastic model of DNA methylation (Jeltsch and Jurkowska, 2014). Such epigenomic changes may lead to tissue dysfunctions in later life. For example, there may be a link between the epigenetic deregulation caused by ART procedures in embryonic liver and blastomere biopsy-induced changes in hepatic gene expression profile and/or function, which we and others observed (Gu et al., 2018; Zacchini et al., unpublished). The epigenetic alterations observed in placental tissues may be indicative of impaired in utero environment, which could contribute to the onset of physiological, metabolic and/or transcriptional changes in the adult offspring (Feuer and Rinaudo, 2017).

Even if differences in physiology and metabolism must be considered before translating animal data to humans, data obtained from different animal models will provide useful information for defining tools and markers that can be applied in human research and, later, create a safe environment for the application of ART. It is generally assumed that ART-induced epigenetic changes are persistent and confer the associated disease risks, i.e. by altering developmental pathways. However, so far we cannot exclude the possibility that the observed epigenetic changes are only a secondary phenomenon. To directly proof the causal relationship between ART-induced epigenetic alterations and observed medical problems, it will be necessary to experimentally introduce specific epigenetic changes in germ cells and/or early embryos and, thereby, induce a specific disease phenotype. Emerging technologies for editing DNA methylation patterns (Liu et al., 2016) will be essential for providing a functional proof of the epigenetic mechanism underlying ART-associated disease risks.

\section{Conclusion}

There is an urgent need to assess the long-term epigenetic risks and safety of ART, to offer the best therapeutic solutions to the growing number of infertile couples worldwide and to improve our ability to monitor health and prevent and manage disease. Despite significant advances in the field of epigenetics, we are still far from fully understand the real epigenetic risks (if any) of ART in the short, medium and long term.

Author's roles

FZ prepared the manuscript; SS, AMS and TH critically reviewed the content; GEP conceived the idea of the project and edited the final version of the manuscript.

\section{Funding}

This project has received funding from the European Union's Horizon 2020 Research and Innovation Programme under grant agreement No 692185 (Acronym ERAofART), by the National Science Centre of Poland (GA no. 2016/21/B/NZ3/03631 and by KNOW Leading National Research Centre (GAno KNOW/IGHZ/RMK/PhD/2016/07) to GEP and by the National Science Centre of Poland (GA no. 2015/19/D/NZ4/03696) to FZ.

\section{Conflict of interest}

None of the authors have a conflict of interest.

\section{References}

AMATO P, TACHIBANA M, SPARMEN M, MITALIPOV S (2014). Three-Parent IVF: Gene Replacement for the Prevention of Inherited Mitochondrial Diseases. Fertil Steril 101: 31-35.

ANCKAERT E, RYCKE MD, SMITZJ (2013). Culture of oocytes and risk of imprinting defects. Hum Reprod Update 19: 52-66.

BARKER DJ, WINTER PD, OSMOND C, Margetts B, Simmonds SJ (1989). Weight in infancy and death from ischaemic heart disease. Lancet 2: 577-580.

BARLOW DP (2011). Genomic Imprinting: AMammalian Epigenetic Discovery Model. Annu Rev Genet 45: 379-403.

BENNET-BAKER PE, WilLKOWSKI J, BURKE DT (2003). Age-associated activation of epigenetically repressed genes in the mouse. Genetics. 165: 2055-2062.

BERTOLDO MJ, LOCATELLI Y, O'NEILL C, MERMILLOD P (2015). Impacts of and interactions between environmental stress and epigenetic programming during early embryo development. Reprod Fertil Dev 27: 1125-1136.

BLOISE E, FEUER SK, RINAUDO PF (2014). Comparative intrauterine development and placental function of ART concepti: implications for human reproductive medicine and animal breeding. Hum Reprod Update 20: 822-839.

BRISON DR, ROBERTS SA, KIMBER SJ (2013). How should we assess the safety of IVF technologies? Reprod Biomed Online 27: 710-721. 
CANOVAS S, IVANOVAE, ROMARR, GARCIA-MARTINEZS, SORIANO-UBEDAC, GARCIA-VAZQUEZ FA, SAADEH H, ANDREWS S, KELSEY G, COY P (2017). DNA methylation and gene expression changes derived from assisted reproductive technologies can be decreased by reproductive fluids. Elife 6. pii: e23670.

CANTONE I and FISHER AG (2013). Epigenetic programming and reprogramming during development. Nat Struct Mol Biol 20: 282-289.

CHEN M and ZHANG L (2011). Epigenetic mechanisms in developmental programming of adult disease. Drug Discov Today 16: 1007-1018.

CHOUX C, CARMIGNAC V, BRUNO C, SAGOT P, VAIMAN D, FAUQUE P (2015). The placenta: phenotypic and epigenetic modifications induced by Assisted Reproductive Technologies throughout pregnancy. Clin Epigenetics 7: 87.

DONJACOUR A, LIU X, LIN W, SIMBULAN R, RINAUDO PF (2014). In vitro fertilization affects growth and glucose metabolism in a sex-specific manner in an outbred mouse model. Biol Reprod 90: 80.

DUMOULIN JC, LAND JA, VAN MONTFORT AP, NELISSEN EC, COONEN E, DERHAAG JG, SCHREURS IL, DUNSELMAN GA, KESTER AD, GERAEDTS JP, et al., (2010) Effect of in vitro culture of human embryos on birthweight of newborns. Hum Reprod 25: 605-612.

DURANTHON V and CHAVATTE-PALMER P. (2018). Long term effects of ART: What do animals tell us? Molec. Reprod. Dev. 85: 348-368

EL HAJJ N, ZECHNER U, SCHNEIDER E, TRESCH A, GROMOLL J, HAHN T, SCHORSCH M, HAAFT (2011). Methylation status of imprinted genes and repetitive elements in sperm DNA from infertile males. Sex Dev 5: 60-69.

ELHAJJ N and HAAFT (2013). Epigenetic disturbances in in vitrocultured gametes and embryos: implications for human assisted reproduction. Fertil Steril. 99: 632-641.

ELHAJJ N, HAERTLEL, DITTRICHM, DENKS, LEHNEN H, HAHNT, SCHORSCHM, HAAF T (2017). DNA methylation signatures in cord blood of IVF/ICSI children. Hum Reprod 32: 1761-1769.

EL-MAARI O, BUITING K, PEERY EG, KROISEL PM, BALABAN B, WAGNER K, URMAN B, HEYD J, LICH C, BRANNAN CI, WALTER J, HORSTHEMKE B (2001). Maternal methylation imprints on human chromosome 15 are established during or after fertilization. Nat Genet. 27: 341-344.

FAZZARI MJ and GREALLY JM (2010). Statistical Methods in Molecular Biology. Stat Methods Mol Biol Methods Mol Biol (Clifton, NJ) 620: 243-265.

FEUER S, CAMARANO L, RINAUDO PF (2013). ART and health: clinical outcomes and insights on molecular mechanisms from rodent studies. Mol Hum Reprod 19: 189-204.

FEUER S and RINAUDO P (2016) From Embryos to Adults: A DOHaD Perspective on In vitro Fertilization and Other Assisted Reproductive Technologies, Healthcare (Basel) 4: 51.

FEUER S and RINAUDO PF (2017). Physiological, metabolic and transcriptional postnatal phenotypes of in vitro fertilization (IVF) in the mouse. J Dev Orig Health Dis. 8: 403-410.

GLUCKMAN PD, HANSON MA, BUKLIJAS T, LOW FM, BEEDLE AS (2009) Epigenetic mechanisms that underpin metabolic and cardiovascular diseases. Nat Rev Endocrinol. 5: 401-408.

GRECO E, BIRICIK A, COTARELO RP, IAMMARONE E, RUBINO P, TESARIK J, FIORENTINO F, MINASI MG (2015). Successful implantation and live birth of a healthy boy after triple biopsy and double vitrification of oocyte-embryo-blastocyst. Springerplus 4: 22.

GU L, ZHANG J, ZHENG M, DONG G, XU J, ZHANG W, WU Y, YANG Y, ZHU H (2018). A potential high risk for fatty liver disease was found in mice generated after assisted reproductive techniques. J Cell Biochem 119: 1899-1910.

HARPER J, GERAEDTS J, BORRY P, CORNEL MC, DONDORP WJ, GIANAROLI L, HARTON G, MILACHICH T, KAARIAINEN H, LIEBAERS I, et al., (2014). Current issues in medically assisted reproduction and genetics in Europe: Research, clinical practice, ethics, legal issues and policy. Hum Reprod 29: 1603-1609.

HARPER J, WELLS D, SIMPSON JL (2015). Controversies in prenatal diagnosis 4: Preimplantation genetic screening should be routinely offered to all preimplantation genetic diagnosis cases. Prenat Diagn 36: 25-28.

HEINDELL JJ and VANDENBERG LN (2015). Developmental Origins of Health and Disease: A Paradigm for Understanding Disease Etiology and Prevention. Curr. Opin. Pediatrics. 27: 248-253

HEMKEYER SA, SCHWARZER C, BOIANI M, EHMCKE J, LE GAC S, SCHLATT S, NORDHOFF V (2014). Effects of embryo culture media do not persist after implantation: a histological study in mice. Hum Reprod. 29: 220-233.

HORSTHEMKE B (2018). A critical view on transgenerational epigenetic inheritance in humans. Nat Commun 9: 2973.

JIANG Z, WANG Y, LIN J, XU J, DING G, HUANG H (2017). Genetic and epigenetic risks of assisted reproduction. Best Pract Res Clin Obstet Gynaecol. 44: 90-104.

JELTSCHA and JURKOWSKARZ (2014). New concepts in DNA methylation. Trends Biochem Sci. 39: 310-318.

JENKINS TG, ASTON KI, PFLUEGER C, CAIRNS BR, CARRELL DT (2014). Ageassociated sperm DNA methylation alterations: possible implications in offspring disease susceptibility. PloS Genet 10: e1004458.

KOBAYASHI H, HIURA H, JOHN RM, SATO A, OTSU E, KOBAYASHI N, SUZUKI R, SUZUKI F, HAYASHI C, UTSUNOMIYA T, YAEGASHI N, ARIMA T (2009). DNA methylation errors at imprinted loci after assisted conception originate in the parental sperm. Eur J Hum Genet 17: 1582-1591.

LIU XS, WU H, JIX, STELZERY, WU X, CZAUDERNAS, SHU J, DADON D, YOUNG RA, JAENISCH R (2016) Editing DNA Methylation in the Mammalian Genome. Cell. 167: 233-247.e17.

MARQUES CJ, COSTA P, VAZ B, CARVALHO F, FERNANDES S, BARROS A, SOUSA M (2008). Abnormal methylation of imprinted genes in human sperm is associated with oligozoospermia. Mol Hum Reprod 14: 67-74.

MARKET-VELKER BA, FERNANDES AD, MANN MRW (2010). Side-by-Side Comparison of Five Commercial Media Systems in a Mouse Model: Suboptimal In vitro Culture Interferes with Imprint Maintenance. Biol Reprod 83: 938-950.

MEISTER TA, RIMOLDI SF, SORIA R, VON ARX R, MESSERLI FH, SARTORI C, SCHERRER U, REXHAJ E (2018) Association of Assisted Reproductive Technologies With Arterial Hypertension During Adolescence. J Am Coll Cardiol 72: 1267-1274.

MOORE SE (2016). Early life nutritional programming of health and disease in The Gambia. J Dev Orig Health Dis 7: 123-131.

NELISSENECM, DUMOULINJCM, DAUNAYA, EVERS JLH, TOST J, MONTFOORT AP (2013). Placentas from pregnancies conceived by IVF/ICSI have a reduced DNA methylation level at the $\mathrm{H} 19$ and MEST differentially methylated regions. Hum Reprod 28: 1117-1126.

ODOM LN and SEGARS J (2011). Imprinting disorders and assisted reproductive technologies. Curr Opin Endocrinol Diabetes Obes. 18: 1492-1501.

POTABATTULA R, DITTRICH M, BÖCK J, HAERTLE L, MÜLLER T, HAHN T, SCHORSCH M, EL HAJJ N, HAAF T (2018). Allele-specific methylation of imprinted genes in fetal cord blood is influenced by cis-acting genetic variants and parental factors. Epigenomics 10: 1315-1326.

PTAK GE, D'AGOSTINO A, TOSCHI P, FIDANZAA, ZACCHINI F, CZERNIK M, MONACO F, LOI P (2013). Post-implantation mortality of in vitro produced embryos is associated with DNA methyltransferase 1 dysfunction in sheep placenta. Hum Reprod 28: 298-305.

REIK W, DEAN W, WALTER J (2011a) Epigenetic reprogramming in mammalian development. Science 293: 1089-1093.

REIK W and DEAN W (2001b). DNA methylation and mammalian epigenetics. Electrophoresis 22: 2838-2843.

RINAUDO P and SCHULTZ RM (2004). Effects of embryo culture on global pattern of gene expression in preimplantation mouse embryos. Reproduction 128: 301-311.

ROSE CM, VAN DEN DRIESCHE S, MEEHAN RR, DRAKE AJ (2013). Epigenetic reprogramming: preparing the epigenome for the next generation. Biochem. Soc. Trans. 41: 809-814.

ROSEBOOM T, DE ROOIJ S, PAINTER R (2006). The Dutch famine and its long-term consequences for adult health. Early Hum Dev. 82: 485-491.

SADAKIERSKA-CHUDYAand FILIPMA (2014) Comprehensive View of the Epigenetic Landscape. Part II: Histone Post-translational Modification, Nucleosome Level, and Chromatin Regulation by ncRNAs. Neurotox Res 27: 172-197.

SCHWARZER C, ESTEVS TC, ARAUZO-BRAVO MJ, LE GAC S, NORDHOFF V SCHLATT S, BOIANI M (2012). ART culture conditions change the probability of mouse embryo gestation through defined cellular and molecular responses. Hum Reprod. 27: 2627-2640.

SMALLWOOD SA and KELSEY G (2012). De novo DNA methylation: a germ cell perspective. Trends Genet. 28: 33-42.

SZYF M (2015). Nongenetic inheritance and transgenerational epigenetics. Trends Mol Med 21: 134-144. 
VAN MONTFOORT APA, HANSSEN LLP, DE SUTTER P, VIVILLE S, GERAEDTS JPM, DE BOER P (2012). Assisted reproduction treatment and epigenetic inheritance. Hum Reprod Update 18: 171-197.

VROOMAN LA and BARTOLOMEI MS (2017). Can assisted reproductive technologies cause adult-onset disease? Evidence from human and mouse. Reprod Toxicol. 68: 72-84.
ZACCHINI F, STANKIEWICZ AM, BERNHARD L, HAAF T, PTAK GE (2018). Blastomere Biopsy: how it affects further development. 2018, Conference procceedings - International Conference "Assisted Reproductive Techlogies: long term perspectives", Cracow, 7-8 September 2018(Abstr).

ZHU J, ZHUANG X, CHEN L, LIU P, QIAO J (2014). Effect of embryo culture media on percentage of males at birth. Hum Reprod 30: 1039-1045. 


\section{Further Related Reading, published previously in the Int. J. Dev. Biol.}

Could modifications of signalling pathways activated after ICSI induce a potential risk of epigenetic defects?

Brigitte Ciapa and Christophe Arnoult

Int. J. Dev. Biol. (2011) 55: 143-152

https://doi.org/10.1387/ijdb.103122bc

The effect of superovulation on the contributions of individual blastomeres from 2-cell stage CF1 mouse embryos to the blastocyst Mika Katayama and R. Michael Roberts

Int. J. Dev. Biol. (2010) 54: 675-681

https://doi.org/10.1387/ijdb.092942mk

Preimplantation genetic diagnosis and embryo research--human developmental biology in clinical practice P Braude

Int. J. Dev. Biol. (2001) 45: 607-611

http://www.intjdevbiol.com/web/paper/11417905

Legal, ethical and historical aspects of assisted human reproduction

C R Austin

Int. J. Dev. Biol. (1997) 41: 263-265

http://www.intjdevbiol.com/web/paper/9184333

Recent scientific and medical advances in assisted human conception

R G Edwards

Int. J. Dev. Biol. (1997) 41: 255-262

http://www.intjdevbiol.com/web/paper/9184332
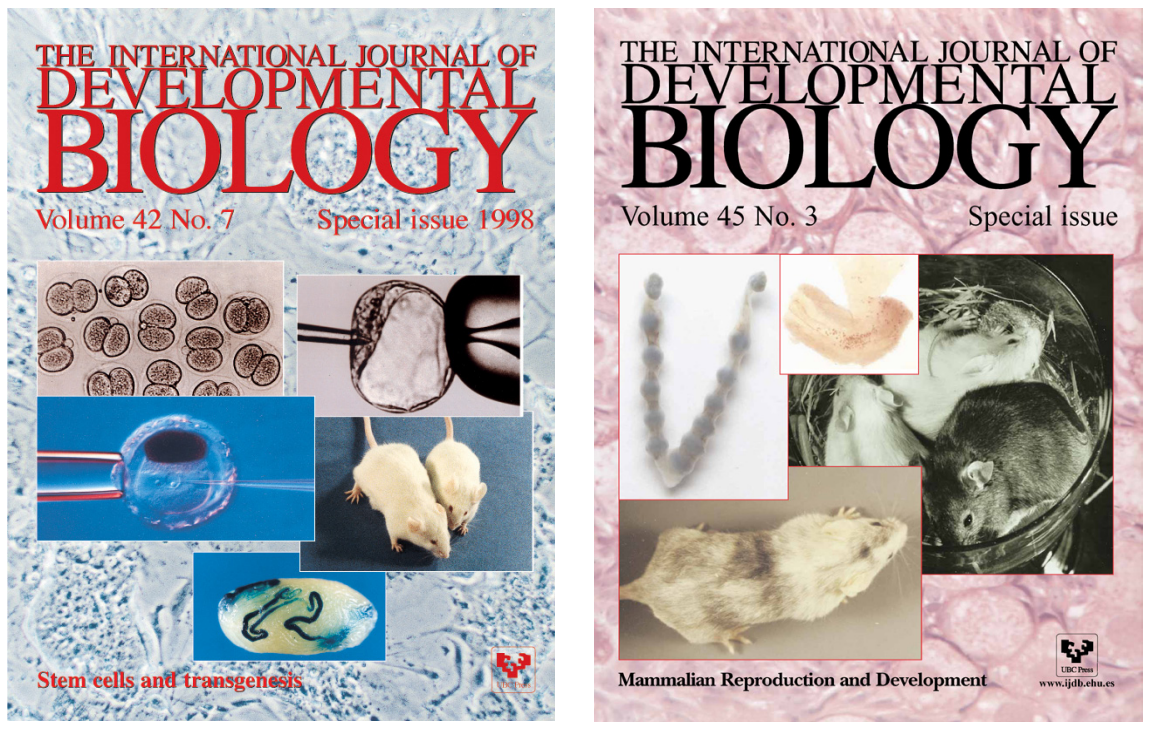



Volume 52 Nos. $2 / 3$ 2008

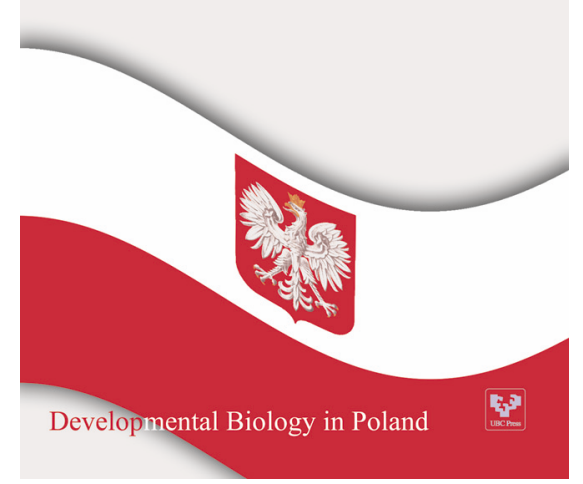

